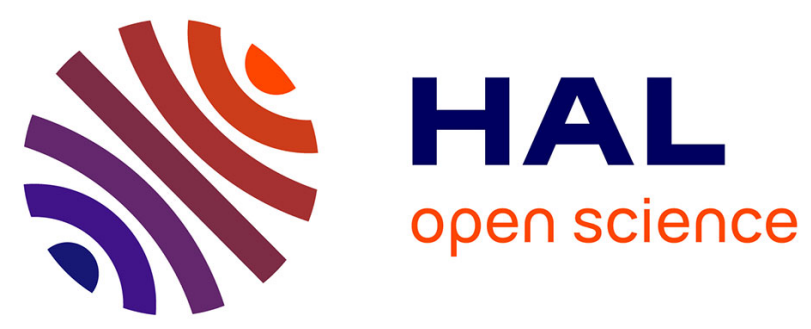

\title{
ÉTUDE ANALYTIQUE D'UN FAISCEAU ACOUSTIQUE BORNÉ EN RÉGIME IMPULSIONNEL
}

M Rousseau, Ph. Gatignol

\section{- To cite this version:}

M Rousseau, Ph. Gatignol. ÉTUDE ANALYTIQUE D'UN FAISCEAU ACOUSTIQUE BORNÉ EN RÉGIME IMPULSIONNEL. Journal de Physique IV Proceedings, 1992, 02 (C1), pp.C1-717-C1-720. 10.1051/jp4:19921155 . jpa-00251114

HAL Id: jpa-00251114

https://hal.science/jpa-00251114

Submitted on 1 Jan 1992

HAL is a multi-disciplinary open access archive for the deposit and dissemination of scientific research documents, whether they are published or not. The documents may come from teaching and research institutions in France or abroad, or from public or private research centers.
L'archive ouverte pluridisciplinaire HAL, est destinée au dépôt et à la diffusion de documents scientifiques de niveau recherche, publiés ou non, émanant des établissements d'enseignement et de recherche français ou étrangers, des laboratoires publics ou privés. 


\title{
ÉTUDE ANALYTIQUE D'UN FAISCEAU ACOUSTIQUE BORNÉ EN RÉGIME IMPULSIONNEL
}

\author{
M. ROUSSEAU et Ph. GATIGNOL* \\ Université Paris VI, Laboratoire de Modélisation en Mécanique associé au CNRS URA 229, Tour 66, \\ 4 place Jussieu, F-75252 Paris cedex 05, France \\ *Université de Technologie de Compiègne, Groupe DAVI, BP. 649, F-60206 Compiègne, France
}

\begin{abstract}
:
In this paper, we propose an analytical study of the acoustical beam produced by a source of finite size, emitting, in a two-dimensional fluid medium, an arbitrary signal, with oblique incidence.

The pressure field, given by a LAPLACE - FOURIER transform, can be written, by means of the CAGNIARD - DE HOOP method, as a single convolution showing that the spatial and temporal effects are uncoupled.

The solution so obtained depends on the incidence of the beam, with the introduction of a time delay.

The spatial component of the reponse depends on the normal velocity data on the plane source - two different distributions are then considered.
\end{abstract}

\section{Introduction.}

Les techniques expérimentales ultrasonores utilisent des émetteurs de petite taille qui émettent des signaux impulsionnels plus ou moins courts. Les phénomènes de diffraction qui apparaissent lors de la propagation de faisceaux bornés en régime monochromatique ou le comportement des signaux impulsionnels émis par des sources ponctuelles ont été largement étudiés au cours de ces dernières années $([1],[2],[3],[4])$.

Par contre, l'étude des champs créés par des émetteurs bornés fonctionnant en régime impulsionnel a été très peu abordée. Nous pouvons distinguer essentiellement deux approches. La première consiste à décomposer la surface de l'émetteur en sources ponctuelles (Fonction de GREEN) et à effectuer une transformation de FOURIER en temps, pour aboutir à l'intégrale de RAYLEIGH, étudiée par STEPANISHEN [5], TJØITA [6], HARRIS [7], pour différentes configurations de source et pour divers profils de faisceau. La deuxième approche consiste à représenter la solution par une double transformée de LAPLACE (en temps) et de FOURIER (en espace - décomposition en ondes planes). Cette dêmarche a été utilisêe par BRESSE et HUTCHINS [8] en 1989 pour une source à symétrie circulaire, émettant sous incidence normale à la surface d'un solide élastique. La forme intégrale de la solution, obtenue par inversion de CAGNIARD DE HOOP, se prète à un traitement numérique plus performant que celle utilisant la fonction de GREEN (absence de bruit numérique). 
L'étude que nous envisageons ici est motivée par les résultats de BRESSE et HUTCHINS qui nous confortent dans le bien-fondé à vouloir systématiser l'utilisation de la méthode de CAGNIARD - DE HOOP, pour étudier les effets de réflexion et de transmission sur des interfaces élémentaires ou des milieux stratifiés (périodiques ou non). Pour ce faire, il nous a semblé indispensable, dans un premier temps, de nous consacrer à l'étude du champ incident dans un milieu fluide parfait. Dans le cas bi-dimensionnel, le faisceau émis sous incidence oblique, s'écrit come le produit de convolution de deux fonctions : l'une dépendant de la forme du signal (réponse temporelle) et l'autre du profil d'amplitude et de la géométrie de la source (réponse spatiale). Alors que, dans les travaux de BRESSE et HUTCHINS, la réponse spatiale est connue sous forme intégrale, l'étude en 2D permet d'expliciter la solution à 1 'aide d'une fonction élémentaire décrivant les effets de diffraction et $1^{\prime}$ influence de $1^{\prime}$ angle d'émission par $1^{\prime}$ intermédiaire d'un temps retardé. Pour une géométrie donnée, sa forme dépend du choix du profil d'amplitude à la source; nous détaillerons 1 'étude $d^{\prime}$ un mode piston et mentionnerons les résultats relatifs à un autre type de profil.

\section{Expression du champ de pression.}

On considère un milieu fluide, parfait, caractérisé par sa masse volumique $\rho$ et la vitesse du son $c$.

Le problème est bi-dimensionnel, dans le plan $(x, z)$, et 1 'émetteur est placé dans le fluide, en $\mathrm{z}=0$.

La vitesse normale sur l'émetteur est donnée par :

$$
V_{n}(x, o, t)=V_{0} f\left(t-t_{r}(x)\right) H\left(t-t_{r}(x)\right) g(x)
$$

avec ${ }^{\circ} f(t) \rightarrow 0$ quand $t \rightarrow+\infty$

- $t_{r}(x)=\sin \theta_{1} x / c$

L'expression (1) modélise 1 'émission, sous incidence oblique $\theta_{1}$, d'un faisceau de profil $g(x)$; le signal de forme quelconque $f(t)$, étant émis sur une durée limitée.

Dans ces conditions, la transformée de LAPLACE du champ de pression émis dans le fluide s'écrit :

$$
\bar{p}(x, z, s)=\frac{\rho V_{0} C \bar{f}(s)}{2 \pi} \int_{-\infty}^{+\infty} \frac{\bar{G}\left(\bar{k}_{x}\right)}{\bar{k}_{z}} e^{s\left(1 \bar{k}_{x} x-\bar{k}_{z} z\right) / c} d \bar{k}_{x}
$$

où $\cdot \bar{k}_{x}=k_{x} /(s \mid c)$ est la variable d'intégration sans dimension,

- $\overline{\mathbf{k}}_{\mathbf{z}}^{2}=1+\overline{\mathbf{k}}_{\mathbf{x}}^{2}$ est une fonction multiforme de $\overline{\mathbf{k}}_{\mathbf{x}}$ dont la détermination est choisie pour vêrifier : $\operatorname{Imk}_{z}<0$ (ondes propagatives dans le sens $z>0$ )

: Re $\bar{k}_{z}>0$ (ondes évanescentes dans le sens $z>0$ ),

- $\bar{f}$ est la transformée de LAPLACE de $f$. 


$$
\bar{G}\left(\bar{k}_{x}\right)=\frac{s}{c} \int_{-\infty}^{+\infty} g(x) e^{-s t} r(x) e^{-1 s \bar{k}_{x}(x \mid c)} d x
$$

3. La méthode de CAGNIARD - DE HOOP.

Pour certaines données $g(x), 1$ 'intégrale (2) peut être résolue par la méthode de CAGNIARD - DE HOOP. Le principe est de trouver, dans le plan complexe de la variable $\bar{k}_{x}$, un contour $C$ équivalent au contour initial réel, le long duquel, on a :

$$
\overline{\mathrm{P}}(\mathrm{x}, z, \mathrm{~s})=\frac{\rho \operatorname{VoC} \overline{\mathrm{f}}(\mathrm{s})}{2 \Pi} \iint_{C} \mathrm{~F}\left(\overline{\mathrm{k}}_{\mathrm{x}}\right) \mathrm{e}^{-\operatorname{sh}\left(\overline{\mathrm{k}}_{\mathrm{x}}\right)} d \overline{\mathrm{k}}_{\mathrm{x}}
$$

avec $h\left(\bar{k}_{x}\right)=t$ réel positif lorsque $\bar{k}_{x}$ décrit $c$

Alors,

$$
\bar{P}(x, z, s)=\frac{\rho \operatorname{Voc} \bar{f}(s)}{2 \pi} \int_{0}^{+\infty} \mathrm{P}_{1 \mathrm{mp}}(x, z, t) e^{-s t} d t
$$

et par identification,

$$
P(x, z, t)=\frac{\rho \operatorname{VoC}}{2 \pi} f(t) o P_{1 m p}(x, z, t)
$$

où $P_{1 \mathrm{mp}}(x, z, t)$ est la réponse impulsionnelle (f $(t)=\delta(t)$ ).

L'expression (6) montre que $P(x, z, t)$ s'écrit comme le produit de convolution de deux fonctions élémentaires mettant en évidence le découplage entre la donnée temporelle $f(t)$ et la donnée spatiale contenue dans $P_{\text {imp }}(x, z, t)$.

\section{Application au cas d'une source piston.}

La fonction $\mathrm{g}(\mathrm{x})$ est alors définie par :

$$
g(x)=(H(x+a)-H(x-a))
$$

ou $\mathrm{H}$ est la fonction de Heaviside, donnée par :

$H(x)=(1$ si $x>0$, o si $x<0,1 / 2$ si $x=0)$

Par application de la méthode de CAGNIARD - DE HOOP, nous obtenons :

$$
\begin{aligned}
P(x, z, t) & =\frac{\rho V_{o} C}{\cos \theta_{1}} f\left(t-t_{R e t}\right) H\left(t-t_{R e t}\right)\left(H\left(x+a-z \operatorname{tg} \theta_{i}\right)-H\left(x-a-z \operatorname{tg} \theta_{i}\right)\right) \\
& +\frac{\rho V_{0} C}{2 \Pi} f(t) \circ\left(P_{i m p(-a)}(t) H\left(t-t_{(-a)}\right)-P_{i m p(a)}(t) H\left(t-t_{(a)}\right)\right)
\end{aligned}
$$


Le premier terme correspond au faisceau direct, émis au sens de 1'acoustique géométrique, entre les deux droites d'équation :

$$
x= \pm a+z \operatorname{tg} \theta_{i}, 0 \leqslant \theta_{i}<\pi / 2
$$

Le deuxième terme modélise les effets de diffraction

$$
P_{i m p(u)}(t)=\frac{2 r_{(u)} C\left(r_{(u)} \sin \theta_{1}-C\left(t-t_{r}(u)\right) \sin \theta_{(u)}\right)}{D}
$$

avec

$$
D=\left(\left[c^{2}\left(t-t_{r}(u)\right)^{2}-r_{(u)}^{2}\right]^{1 / 2} x\right.
$$

$$
\left.\left[\left(r_{(u)} \sin \theta_{1}-c\left(t-t_{r}(u)\right) \sin \theta_{(u)}\right)^{2}+\cos ^{2} \theta_{(u)}\left(c^{2}\left(t-t_{r}(u)\right)^{2}-r_{(u)}^{2}\right)\right]\right)
$$

représente la contribution du bord $x=u$ de l'émetteur, et :

$$
t_{(u)}=\frac{\sin \theta_{i} u+r_{(u)}}{C}
$$

est le temps retardé correspondant, avec :

$$
r_{(u)}=\left((x-u)^{2}+z^{2}\right)^{1 / 2}, \sin \theta_{(u)}=\frac{x-u}{r_{(u)}}, \cos \theta_{(u)}=\frac{z}{r_{(u)}}
$$

\section{Remarque :}

La méthode de CAGNIARD - DE HOOP ne peut être appliquée dans le cas d'un faisceau gaussien pour lequel $g(x)=e^{-(x / a)^{2}}$

Pour pallier cette limitation, il est possible d'étudier des profils de la forme :

$$
g(x)=\sum_{n=0}^{2 P+1}(-1)^{n}(x / a)^{2 n} / n !(H(x+a)-H(x-a)) \quad P \text { entier } \geqslant 0
$$

Nous prêsenterons au cours de l'exposé le cas $\mathrm{P}=0$.

\section{Références :}

[1] ROUSSEAU, M. ,GATIGNOL,Ph., J.Acoust.Soc.Am. 78.5 (1985) 1859

[2] CLAYES, J.M. , LEROY, O., J.Acoust. Soc.Am. 72 (1982) 585

[3] TYGEL, M. , HUBRAL, P., Geophysics 50 (1986) 1466

[4] DE HOOP, A.T., VAN DER HIJDEN, J.H.M.T., J.Acoust.Soc.Am. 75.6 (1984) 1709

[5] STEPANISHEN,P.R., J.Acoust.SoC.Am. 45.5 (1971) 1629

[6] TJøITA, J.N. TJøTTA,S., J.Acoust.Soc.Am. 71.4 (1982) 824

[7] HARRIS, G.R., J.Acoust.Soc.Am. 70.1 (1981) 186

[8] BRESSE, L.F., HUTCHINS, D.A., J.Acoust.Soc.Am. 86.2 (1989) 810 